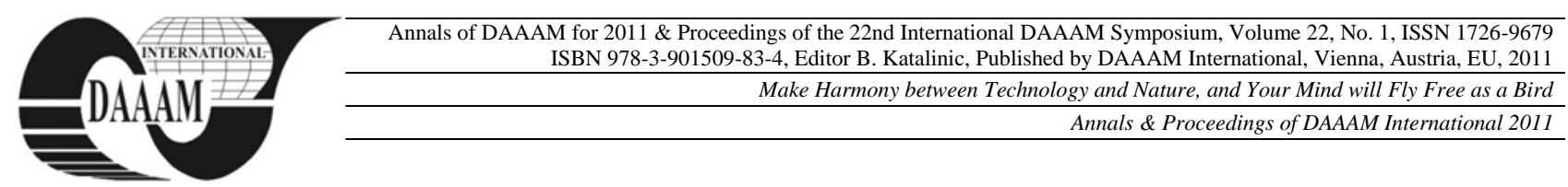

\title{
ANALYSIS OF STRUCTURAL DAMAGES IN SHIP COLLISIONS BASED ON SIMPLIFIED METHOD
}

\author{
DUMITRACHE, C[osmin] L[aurentiu]; DUMITRACHE, R[amona]; CHIRCOR, M[ihael] \\ \& VARSAMI, A[nastasia] E[lena]
}

\begin{abstract}
This paper is part of a procedure which analyses ships collisions, addressing to all types of ships and damage scenarios. The analysis of a ship-ship collision is usually separated into two classes. These are external and internal mechanics. Large plastic deformation of the shell plating subjected to various loadings is developed. Therein is described the work principle for upper-bound theorem and an example for calculating crushing and tearing of a plate.

Key words: collision, simplified method, energy rate, plate deformation
\end{abstract}

\section{INTRODUCTION}

Ships' side structure is a complex one and the deformation, destroying and crushing of the side structures is also. To analyze a structural damage, one of the following methods can be used: empirical method, finite element method, experimental method and simplified method.

Finite element method and simplified method, known also as "super-elements method" are known as theoretical methods for predicting damages. The experimental methods are involving far too big amount of money in order to be used for academically reasons. The finite elements can give detailed solutions, but it requires certain computer power and is still quite expensive.

Of all these, we focused on simplified method, which is based on the upper-bound theorem. Within the simplified analytical method is considered that different structural members, such as side shell, decks and frames, do not interact, but have an independent contribution on the total collision resistance.

The upper-bound method was used before by others, like Wierzbicki (1983, 1993), Abramowicz (1994), Amdahl (1983), Paik \& Pedersen (1995), Simonsen (1997), to analyse the damages of the ship following a collision or grounding and the results were quite close to the experimental ones.

\section{SIMPLIFIED METHOD}

The simplified method is widely used in engineering analysis and design. It has been proved that the method is valuable for estimating the collapse load of a structure subject to extreme loads. The collapse load so obtained can be used as a realistic basis for design. It should be emphasised that the limit analysis is an approximate method. A basic assumption is that the material is perfectly plastic without strain hardening or softening(Zhang, 1999).

The work principle may be expressed by the formula:

$$
\int_{A} F_{i} u_{i} d A+\int_{V} T_{i} u_{i} d V=\int_{V} \sigma_{i j} \varepsilon_{i j} d V
$$

where $\mathrm{A}$ is the surface area, $\mathrm{V}$ is the volume of the structure, $F_{i}$ and $T_{i}$ are the external force and the body force, $\sigma_{i j}$ is any set of stresses, $\varepsilon_{i j}$ is the strain field, $u_{i}$ is the displacement (Pedersen,1993).
The rate form of the virtual work equation is:

$$
\int_{A} F_{i} u_{i}^{\prime} d A+\int_{V} T_{i} u_{i}^{\prime} d V=\int_{V} \sigma_{i j} \varepsilon_{i j}^{\prime} d V
$$

Lower-bound theorem can be expressed as follows: if any system of generalized stresses can be found throughout a structure which is in equilibrium with the applied loads and which nowhere violates the yield condition, then the structure will not collapse or be at the point of collapse. Referring to the upper-bound theorem, we can say that if the work rate of a system of applied loads during any cinematically admissible collapse of the structure is equated to the corresponding internal energy dissipation rate, then the system of loads will cause collapse or be at the point of collapse. The two bound theorems can be used independently. If the calculated loads coincide in the two methods, the exact solution is found (Zhang, 1999).

$$
F \cdot \delta^{\prime}=E_{\text {int }}^{\prime}
$$

This expresses the equilibrium of the external and internal energy, where $F$ is the external force, $\delta$ is the velocity at the force action point and $E_{\text {int }}$ the internal energy rate.

The internal energy rate can be also expressed as:

$$
E_{\mathrm{int}}^{\prime}=\int \sigma_{i j} \varepsilon_{i j}^{\prime} d V
$$

where $\varepsilon_{i j}$ is the rate of strain sensor and $V$ is the volume of the solid body. gives:

For a plane stress condition, the von Mises yield condition

$$
\sigma_{x x}^{2}+\sigma_{y y}^{2}-\sigma_{x x} \sigma_{y y}+3 \sigma_{x y}^{2}=\sigma_{0}^{2}
$$

For a deforming plate, the rate of internal plastic energy dissipation can be written as the sum of the bending and the membrane energy dissipation rate:

$$
E_{\text {int }}^{\prime}=E_{b}^{\prime}+E_{m}^{\prime}
$$

The bending energy rate can be expressed as:

$$
E_{b}^{\prime}=\int_{A} M_{\alpha \beta} k_{\alpha \beta}^{\prime} d A+\sum_{i=1}^{n} M_{0 i} \theta_{i}^{\prime} l_{i}, \quad(\alpha, \beta=1,2)
$$

where $\mathrm{A}$ is the plate area, $k_{\alpha \beta}$ is the curvature of the plating, $\Theta_{i}$ and $l_{i}$ are the rotation and the length of the plastic hinge line, $M_{\alpha \beta}$ is the bending tensor moment, $M_{0}$ is the fully plastic bending moment and $t$ is the plate thickness. 
By use of von Mises yield criterion, the membrane energy rate can be expressed as:

$$
E_{m}^{\prime}=\frac{2}{\sqrt{3}} \sigma_{0} t \int_{A} \sqrt{\varepsilon_{x x}^{\prime 2}+\varepsilon_{y y}^{\prime 2}+\varepsilon_{x x}^{\prime} \varepsilon_{y y}^{\prime}+\varepsilon_{x y}^{\prime 2}} d x d y
$$

\section{IN-PLANE CRUSHING AND TEARING OF PLATES}

In-plane crushing and tearing of plates is one of the superelements on which internal mechanics models are based on. Altogether, they contain solutions for behavior under deep collapse of the assembly. By summing the forces of each superelement is possible to find out the total amount of absorbed energy between the vessels involved.

Crushing of plates can be idealized by simple geometrical modes of deformation. Hereby is used a model suggested by Simonsen and Ocakli (1999) is used. When a deck is loaded by a point load, it will first collapse plastically with folds extending to the nearest boundaries. After a certain penetration, the plate will fracture and it will continue to fold up in front of the bow like a concertina (Lutzen, 2001).

A collision can occur in many places around the vessels and can involve a lot of types of plates, as following:

- the bottom and the inner bottom, the stringer decks and the stringers, main deck, intermediate decks, are considered horizontal plates

- the floors, the girders, the frames (simple or web) and transverse bulkheads are considered vertical plates.

Transverse stiffeners, stiffeners parallel to the direction of penetration, are crushed axially. Longitudinal stiffeners are included in the model by smearing out the volume of the stiffener in the longitudinal direction, resulting in an orthotropic plate (Lutzen, 2001).

The load-deformation relation can be expressed, as per Simonsen and Ocakli, by:

$$
\begin{gathered}
P=1.56 \sigma_{0} t^{5 / 3} \frac{b_{1}+b_{2}}{\left(b_{1} b_{2}\right)^{1 / 3}}+0.518 \sigma_{0} t_{e q}^{4 / 3} \frac{b_{1}+b_{2}}{\left(b_{1} b_{2}\right)^{2 / 3}} . \\
\cdot\left[1+\frac{w}{1.51\left(b_{1} b_{2} t\right)^{1 / 3}}\right] \mathrm{f}(w)
\end{gathered}
$$

where $w$ is the deflection, $t$ is the thickness of the plate. The thickness is corrected for every new involved longitudinal, which volume is smeared out to give the equivalent thickness $t_{e q}$. The involved function, $f$, depends on the depth of the plate, which is defined as:

$$
f(w)= \begin{cases}w\left(1+\frac{w}{4 H}\right) & \text { for } w \leq D \\ D\left(1+\frac{w}{2 H}-\frac{D}{4 H}\right) & \text { for } w>D\end{cases}
$$

where $D$ is the depth of the plate and $H$ is the folding length, which can be determined as follows:

$$
H=0.377 \sqrt[3]{b_{1} b_{2} t}
$$

After rupture, the folding mode changes to concertina tearing. Wierzbicki (1995) found that concertina tearing can be expressed as:

$$
P_{m}=4.33 \sigma_{0} t^{5 / 3}\left(\frac{b_{1}+b_{2}}{2}\right)^{2}+\frac{8}{3} R_{c} t
$$

where $R_{c}$ is the fracture toughness. The fracture toughness for mild steel is in the range $R_{c}=300-1000 \mathrm{~N} / \mathrm{mm}$.

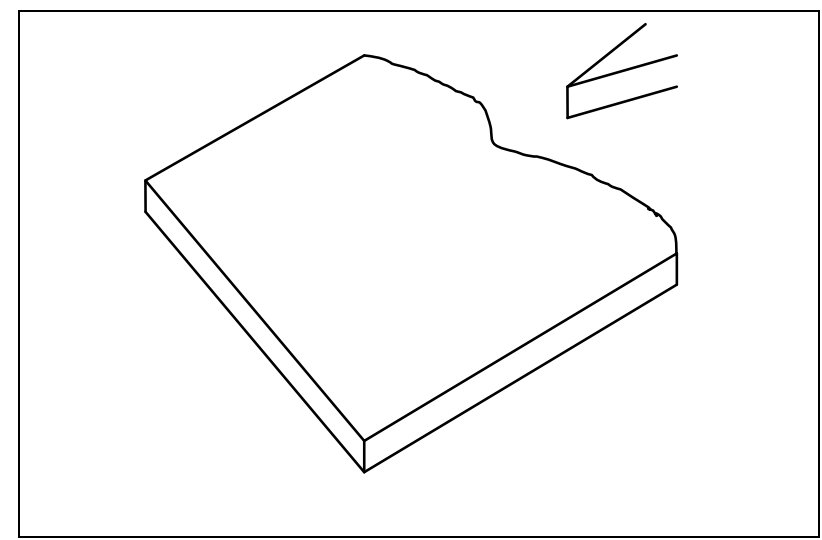

Fig. 1. In-plane plate deformation

\section{CONCLUSIONS}

The presentation is part of a procedure which analyses ships collisions, addressing to all types of ships and damage scenarios, being difficult to obtain a precise calculation of the internal mechanics as the collision is based on a complicated combination of buckling, yielding, tension, tearing, rupture and brittle failure of materials.

The method based on the super-element method, where the ship's structure is separated into its structural elements like plates, beams, or plate intersections like $\mathrm{X}$ and $\mathrm{T}$ elements is a simplified but rational model for determining the internal mechanics. The use of super-element solution calls for adaptive or successive discretisation. By summing up the crushing force of each super-element, it's possible to determine the total contact load between the two involved vessels and the total amount of absorbed energy.

The use of simplified method helps to compare and validate the mathematical approach. Theoretical results are compared with existing simulating experiments. The upper-bound method is used to calculate the structural plastic deformation and energy dissipation under extreme loading and further on, it helps to calculate full-scale ship-ship and ship-various objects collisions.

\section{REFERENCES}

Abramowicz, W. (1994). Crushing Resistance of T, Y and X Sections, MIT-Industry Joint Program on Tanker Safety, Massachusetts Institute of Technology, USA, Report No. 24

Brown, A. (2002). Collision scenarios and probabilistic collision damage. Journal of Marine Structures, Vol. 15, Pages 335-364.

Lutzen, M. (2001). Ship Collision Damages, PhD Thesis, Technical University of Denmark, ISBN 87-89502-60-4

Pedersen, P. T., Valsgaard, S., Olsen, D. and Spangenberg, S. (1993), Ship Impacts: Bow Collisions, International Journal of Impact Engineering, Volume 13, No. 2, Pages 163-187, London

Simonsen, B.C. \& Ocakli, H. (1999). Experiments and Theory on Deck and Girder Crushing, Thin-Walled Structures, Volume 34, Pages 195-216

Wierzbicki, T. (1995). Concertina tearing of metal plates, International Journal of Solids and Structures, Volume 32, No. 19, Pages 2923-2943

Zhang, S. (1999). The Mechanics of Ship Collisions, $P h D$ Thesis, Technical University of Denmark, ISBN 87-8950205-1 